\title{
Comunicación y Percepción del Riesgo por Compuestos Orgánicos Persistentes en Jornaleros Agrícolas de Sonora, México
}

\author{
Beatriz Olivia Camarena Gómez \\ Centro de Investigación en Alimentación y Desarrollo, Hermosillo, México. \\ Email: betica@ciad.mx \\ Candelaria Berenice Ochoa Nogales \\ Centro de Investigación en Alimentación y Desarrollo, Hermosillo, México. \\ Email: bereniceochoan@gmail.com
}

\begin{abstract}
Ana Isabel Valenzuela Quintanar
Centro de Investigación en Alimentación y Desarrollo, Hermosillo, México. Email: aquintanar@ciad.mx
\end{abstract}

\begin{abstract}
Resumen: El propósito de este documento es brindar información para fortalecer acciones que permitan coadyuvar a mitigar la situación de riesgo por presencia de Compuestos Orgánicos Persistentes (COP's). Inicia con la descripción de tales compuestos y entrega datos de estudios que muestran sus efectos adversos en la salud ambiental y humana y dan cuenta de su presencia en Sonora, México. Se destaca entonces la necesidad de orientar a la población en riesgo sobre qué hacer al respecto y algunos de los lineamientos que organismos internacionales recomiendan considerar al diseñar una estrategia de comunicación. En seguimiento a tales sugerencias, se muestran resultados de una encuesta de opinión sobre COP's y riesgos implicados, aplicada a jornaleros/as agrícolas y en base a las necesidades de información que tal población exhibe y, finalmente, se delinea el contenido del programa de comunicación.
\end{abstract}

Palabras clave: Contaminantes Orgánicos Persistentes (COP's), percepción, comunicación, riesgo, jornaleros agrícolas.

\section{Communication and risk perception by POC's in agricultural laborers of Sonora, México}

\begin{abstract}
The purpose of this document is to provide information to strengthen actions that allow to contribute to mitigate the risk situation due to the presence of Persistent Organic Pollutants (POP's). It begins with a description of such compounds and presenting data from studies that show its adverse effects in human and environmental health; besides proving its presence in Sonora, Mexico. Therefore, it's necessary to orientate the population at risk over what to do about it and give some guidelines that international agencies recommend to take into
\end{abstract}


account when designing a communication strategy. In the following up on these suggestions, results of an opinion poll on POP's and implicated risks applied to agricultural laborers are shown, and based in the information needs that such population exhibits, the content of the communication program is outlined.

Key words: Persistent Organic Pollutants (POP's), perception, communication, risk, agricultural laborers.

\section{Comunicação e Percepção do Risco de Poluentes Orgânicos Persistentes em Trabalhadores Agrícolas de Sonora, México}

Resumo: O objetivo deste documento é fornecer informações para fortalecer as ações que ajudarão a reduzir o risco por presença de Poluentes Orgânicos Persistentes (POPs). Começa com uma descrição de tais compostos e proporciona dados de estudos que mostram seus efeitos adversos sobre a saúde humana e ambiental e evidenciam a sua presença em Sonora, México. Salienta-se a necessidade de orientar à população em risco sobre o que fazer a respeito e de algumas das orientações que recomendam as organizações internacionais para desenhar uma estratégia de comunicação. Dando seguimento a estas sugestões, se apresentam os resultados de uma pesquisa de opinião sobre os POPs e os riscos envolvidos, aplicada a trabalhadores/as agrícolas e com base nas necessidades de informação desta população. Finalmente se expõe o conteúdo do programa comunicação.

Palavras-chave: Poluentes Orgânicos Persistentes (POPs), percepção, comunicação, risco, trabalhadores agrícolas.

$* * *$

\section{Introducción}

La presencia de COP's en el estado de Sonora, México se asocia con las prácticas agrícolas que fueron propiciadas por la "Revolución Verde": el uso de semillas mejoradas, el monocultivo y la utilización de agroquímicos, como plaguicidas organoclorados. Desde mediados del siglo pasado hasta nuestros días, quienes promueven la modernización agrícola en esta entidad, han replicado básicamente dichas prácticas, por lo que no debe extrañar la presencia de tales compuestos en las localidades de mayor tradición agrícola (Gómez, 2007; Valenzuela et al. 2008; Proyecto 108092, 2010-12).

De ahí el interés por desarrollar una metodología que permita llevar a cabo el inventario, caracterización y verificación de sitios potencialmente contaminados con COP's en las principales zonas agrícolas del estado de Sonora y por diseñar, además, programas de comunicación que orienten a la población sobre qué hacer al respecto (Valenzuela, et al, 2010). Este documento refiere algunos resultados de tal estudio.

Primero, se presenta una breve descripción de los COP's y datos de algunos estudios que han mostrado su nocivo impacto en la salud ambiental y humana. Después, se presenta un resumen de los esfuerzos realizados 
en distintos foros de tipo multilateral para atender este problema y los compromisos asumidos por México. Se incluyen también resultados de algunas investigaciones que muestran la presencia de COP's en Sonora. Y, en el apartado titulado Sociedad de Riesgo y población vulnerable por COP's, tras destacar que la presencia de $\mathrm{COP}^{\prime}$ s es una manifestación más de la problemática ambiental contemporánea que refuerza la necesidad de conformar una sociedad civil con mayor responsabilidad social y planetaria, se enfatiza la necesidad de diseñar e impulsar programas de comunicación con tal propósito. Se precisan entonces algunos lineamientos que sugieren considerar ciertos organismos internacionales al diseñar dichos programas $y$, en seguimiento a tales recomendaciones, se refieren los principales resultados de una encuesta de opinión sobre los COP's y riesgos asociados que fue aplicada a jornaleros/as agrícolas de Pesqueira, localidad de San Miguel de Horcasitas, Sonora, con el propósito de orientar el contenido de la estrategia de comunicación. Ésta se ajusta precisamente a las necesidades de información que dicha población exhibe en el instrumento aplicado. El propósito de la investigación es aportar información que permitan fortalecer acciones para coadyuvar a mitigar la situación de riesgo por presencia de COP's, como es el caso de los jornaleros/ as que laboran en los campos agrícolas de Sonora.

\section{Contaminantes Orgánicos Persistentes (COP’s)}

Los llamados COP's, son sustancias químicas basadas en carbono, resultado de las emisiones al medio ambiente, generadas principalmente por actividades humanas, vinculadas con procesos industriales, subproductos y plaguicidas (IPEN, 2011). Se sabe que estos compuestos poseen una combinación de características extremadamente peligrosas, son: a) altamente tóxicos; b) persistentes -pueden durar muchos años e incluso décadas antes de degradarse en otras formas menos peligrosas-; c) volátiles -pueden evaporarse y viajar grandes distancias por el aire y el agua-; y d) se acumulan en los tejidos grasos. Por su persistencia y movilidad, es posible encontrarlos prácticamente en cualquier lugar del planeta, incluso en lugares donde nunca han sido utilizados.

Tales características llevan a considerar que los riesgos por COP's en la salud humana y ambiental en la actualidad han alcanzado una dimensión global, sin embargo, falta probar y precisar la magnitud de tal problemática por áreas geográficas específicas a nivel local y regional. También, falta conocer la opinión e información que posee al respecto la población vulnerable considerada en mayor situación de riesgo.

Estudios hechos desde mediados del siglo pasado, mostraron el impacto negativo de los COP's en la salud humana y ambiental. En el medio ambiente, por ejemplo, se les relaciona con la disminución de la población de aves (Carson, 1962), de lagartos (Guillete et al., 1994: 680-688) y de garzas (Ohlendorf, et al. 1993: 176-185), con problemas de reproducción de aves rapaces (se alimentan de peces), adelgazamiento de la cáscara de huevos (Walker et al., 1996); así como con la degradación de los suelos y la 
contaminación de mantos acuíferos En la salud humana, son varios los estudios que muestran esa relación de los COP's con patologías diversas. Están desde alergias e insensibilidad hasta alteraciones del sistema hormonal, afectaciones al sistema inmunológico, daños al sistema nervioso central y periférico (PNUMA 2008), daño neurotóxico (Roberts et al., 2007: 1482; Sagiv et al., 2008: 666-673; PNUMA 2008), artritis reumatoide (Lee et al., 2007: 883-888), apoptosis y daño al ADN (Fernández, et al., 2005), daño genotóxico (Torres y López, 2007: 51-60; Martínez y Gómez, 2007: 185-200), efectos carcinogénicos (PNUMA 2008; Ward et al., 2009: 1007-10013; Xu et al., 2010: 60-66), daños reproductivos (PNUMA 2008; Bellingahm et al., 2009: 1556-1552; Wang et al., 2009: 1244-1249), y diabetes (Codru et al., 2007: 1747-1752; Rantakokko et al., 2009: 807-901), entre otros.

Es por demás evidente la presencia de COP's en casi todos los niveles tróficos, como también lo son sus efectos adversos en una gran variedad de especies y en la salud humana. Al parecer, todo ser vivo está expuesto de manera crónica a concentraciones bajas de dichos contaminantes, ya que se mantienen en el ambiente (aire, agua, suelo), aun cuando hoy esté prohibido su uso. De hecho, se presupone que la presencia actual de COP's deriva de acciones realizadas de manera recurrente en décadas pasadas, por ejemplo, el uso de plaguicidas en diversas actividades y procesos agrícolas, el uso de ciertos compuestos químicos como aditivos o auxiliares en una variedad de aplicaciones industriales, con tóxicos generados como subproductos no intencionales en procesos de combustión (dioxinas, furanos y el hexaclorobenceno) en la quema de basura y/o en incendios involuntarios (Ritter et al., 1995, en Yarto et al., 2003).

Los riesgos que generan los COP's en la salud ambiental y humana son una expresión más de la problemática ambiental contemporánea (Camarena, 2006). Al igual que ésta, la problemática ambiental por la presencia de COP's, se puede caracterizar por ser global, compleja y antropogénica. Global, porque si bien el uso de COP's es localizado, la magnitud y propagación de estos compuestos, asociados a sus propiedades químicas, ha adquirido un alcance planetario; compleja, porque en su origen, desarrollo y posibilidades de solución se involucran intereses económicos, políticos y sociales con posicionamientos muchas veces encontrados que dificultan la toma de acuerdos; y antropogénica, porque también la presencia de COP's y riesgos asociados, se vincula con actividades y procesos productivos que van de la mano con un modelo económico que ha priorizado la ganancia, sobre la salud ambiental y humana.

\section{Los COP's en la Agenda Internacional y participación de México}

Así como la temática ambiental irrumpe en el escenario internacional asociada a los problemas del desarrollo, también la de los COP's y riesgos asociados, ocupa un lugar central en la agenda de trabajo de varios foros 
de ámbito mundial. El alcance global de los riesgos por COP's en la salud ambiental y humana quedó expresado en la Convención de Basilea (1989), en la Convención de Roterdam (1998) y en la de Estocolmo (2001). En la primera se instituye el control transfronterizo de residuos peligrosos en respuesta a la incertidumbre de que los residuos tóxicos de los países desarrollados, fueran enviados para su disposición a países en vías de desarro1lo, también llamados de economías en transición (Hazardous Chemicals Conventions, 2002). En la Convención de Roterdam, el interés fue el procedimiento de consentimiento para el manejo de ciertas sustancias químicas peligrosas y plaguicidas, objeto de comercio internacional. En la Convención de Estocolmo quedó establecido el tratado de las Naciones Unidas que prohíbe o minimiza, según sea el caso, el uso de doce de las sustancias tóxicas más utilizadas en el mundo.

En tales foros se distinguen tres niveles o categorías de COP (Cfr: Pérez-Maldonado et al., 2006: 343-351; PNUMA, 2010: 5): a) Plaguicidas (aldrina, clordano, dieldrina, endrina, heptacloro, hexaclorobenceno, mirex, toxafeno, DDT); b) Productos químicos industriales (bifenilos policlorados (BPC), y hexaclorobenceno); y c) Subproductos (hexaclorobenceno, dibenzo-p-dioxinas y dibenzofuranos policlorados (PCDD / PCDF) y BPC). A estos productos se agregan nueve productos químicos más en la Cuarta Reunión de la Conferencia de las Partes realizada en mayo del 2009 (PNUMA, 2010): a) Plaguicidas (alfa-hexaclorociclohexano, betahexaclorociclohexano, clordecona, lindano, pentaclorobenceno); b) Productos químicos industriales (hexabromobifenilo, éter de hexabromodifenilo y éter de heptabromodifenilo, pentaclorobenceno, sufonato de perfluorooctano, éter de tetrabromodifenilo y éter de pentabromodifenilo); y c) Subproductos (alfa-hexaclorociclohexano, beta-hexaclorociclohexano, pentaclorobenceno). Nótese que algunos se repiten entre categorías.

La investigación que dio pie a este trabajo, se centró sólo en la categoría de plaguicidas: a) Para su eliminación, aldrina, clordano, dieldrina, endrina, heptacloro, hexaclorobenceno, mirex, toxafeno, alfahexaclorociclohexano, beta-hexaclorociclohexano, clordecona, lindano; y b) Para su eliminación y/o restricción, el DDT y el pentaclorobenceno.

México firma y ratifica su participación en los convenios citados (Fernández, A. et al., 2005: 21; PNUMA, 2008): la Convención de Basilea (22-III-1989 y el 22-II-1991) y el Convenio de Estocolmo (23-V-2001 y 10-II2003). Al hacerlo, asume el compromiso de regular, reducir y eliminar el uso de COP's en el territorio nacional, lo cual le exige controlar, supervisar y sancionar ciertas prácticas vinculadas al uso de $\mathrm{COP}^{\prime} \mathrm{s}$ en los sectores agrícola e industrial. Habrá que indagar qué se ha hecho en cada aspecto.

En el Convenio de Estocolmo, el compromiso de legislar y promover programas de intervención para evitar/eliminar el uso de los COP's remite al Plan Nacional de Implementación (PNI). Este es el documento rector que enlista los proyectos y actividades a realizar así como las instituciones involucradas en cada tarea. En México, las instituciones que trabajan en 
ese tenor son: la Secretaría del Medio Ambiente y Recursos Naturales (SEMARNAT), el Instituto Nacional de Ecología (INE) y el Consejo Nacional de Ciencia y Tecnología (CONACYT). En el año 2008, estas tres dependencias convocan a los grupos científicos del país a realizar investigaciones para identificar y medir la presencia de COP's a nivel local y regional; y a complementar esos inventarios-diagnósticos, con el diseño e implementación de programas de comunicación. Los proyectos aprobados fueron publicados dos años después, estableciéndose dos o tres años para el desarrollo de cada investigación.

\section{Algunas evidencias de presencia de Plaguicidas Organoclorados (POC's) en Sonora, México}

Estudios recientes han mostrado presencia de POC's, en suelo de campos cercanos a la localidad de Pesqueira, Sonora; así como en algunos vegetales, en leche materna y en personas que trabajan en dichos campos (Gómez, 2007; Valenzuela et al., 2008; Valenzuela et al., 2012). Las investigaciones no precisan si tal presencia corresponde a su uso reciente o histórico, pero se presume que deriva del uso de tales productos en décadas pasadas porque está prohibido su uso en la actualidad.

El trabajo realizado para identificar y cuantificar el DDT y sus metabolitos en leche materna de mujeres residentes en Pesqueira, encontró que el metabolito p'p -DDE se presentaba en mayor concentración $(9.0 \mu \mathrm{g} /$ $\mathrm{Kg}$ ) y con mayor frecuencia (4 de 6); tal contaminación está por debajo de los límites máximos residuales establecidos pero se destaca por las características de bioacumulación en el ambiente y en los organismos que tienen tales plaguicidas (Gómez, 2007).

La identificación de plaguicidas organoclorados y organofosforados en plasma derivó del estudio realizado a una población de 212 jornaleros elegidos de manera aleatoria en cuatro campos agrícolas de alta producción hortofrutícola de la Costa de Hermosillo, Pesqueira y Guaymas (Valenzuela et al., 2008). Se trabajó con 110 hombres y 102 mujeres y los resultados mostraron que el $57 \%$ de los varones y el $62 \%$ de las féminas presentaban residuos de plaguicidas organoclorados. De ese $57 \%$ correspondiente a los hombres: el $90.5 \%$ presentó DDE, el $0.02 \%$ DDE y TDE, el $0.03 \%$ DDT y aldrín, así también el $0.03 \%$, DDE y lindano. Mientras que del $62 \%$ correspondiente a mujeres: el $96.8 \%$ presento DDE, el $0.05 \%$ DDE y lindano, y $0.02 \%$ DDE, TDE y DDT. La misma investigación consideró el análisis de residuos de plaguicidas organoclorados y organofosforados en agua, suelo, uva, tomate y diferentes variedades de chiles recolectados en los campos en estudio; y los resultados mostraron presencia de residuos de plaguicidas organoclorados (DDE) en el caso del tomate y en las variedades de chile -excepto el chile serrano y morrón naranja-; en los chiles Caribe y Anheim se encontró endrín; en la uva, el agua y el suelo no se detectó presencia de tales residuos. 
En otra investigación (Valenzuela et al., 2010), se tomaron muestras de suelo en dos campos agrícolas de Pesqueira, Sonora en tres niveles de profundidad (superficial, $30 \mathrm{~cm}$ y $60 \mathrm{~cm}$ ) y en cinco coordenadas $(15$ muestras por campo). Los resultados preliminares muestran presencia de endrín en los dos campos a un rango de concentración de $0.003 \mu \mathrm{g} / \mathrm{kg}$ a $7.20 \mu \mathrm{g} / \mathrm{kg}$, valores muy por debajo de los límites máximos de riesgo (40 $\mu \mathrm{g} / \mathrm{kg})(\mathrm{EPA}$, 2011). También se encontró a clordando a una concentración de $1.85 \mu \mathrm{g} / \mathrm{kg}$, valor por debajo del LMP $250 \mu \mathrm{g} / \mathrm{kg}$. Esos resultados -nula presencia DDT y sus metabolitos (DDE y DDD), entre otros-, permiten afirmar que son campos de uso reciente (10 a 15 años) y que en ninguno se ha realizado aplicación de plaguicidas organoclorados a gran escala.

\section{Sociedad del Riesgo y población vulnerable por COP en Sonora}

Los conceptos de riesgo y de peligro regularmente se confunden, pero no son lo mismo. Sus diferencias se han establecido en términos probabilísticos, de exposición y origen de cada fenómeno.

Hay quien señala que el riesgo es la probabilidad cuantitativa de ocurrencia de un efecto en la salud después de que el individuo ha sido expuesto a un peligro en una cantidad específica, esto es el peligro "...es una cualidad de la fuente de daño..." (Yassi, et al., 2000); o también, se le define como la "...probabilidad de daño, si se entra en contacto con esa fuente en determinadas circunstancias" (Echemendía, 2011: 475). Estos autores precisan que los peligros no dependen del hombre, los riesgos sí. En ese sentido, Giddens (2000), precisa que la diferencia entre peligro y riesgo está en las fuentes que los generan: las fuerzas de la naturaleza en el primero (epidemias, hambrunas, sequías, inundaciones, terremotos, etc.) y las acciones humanas impulsadas para controlar y dominar tales peligros en el segundo.

Y enfatiza que en la sociedad actual la situación se complica porque “...se están traspasando los límites que separan los riesgos "pronosticables" de los peligros incontrolables" (Beck, 2007: 52). La problemática ambiental contemporánea en sus diversas manifestaciones locales da cuenta de ello.

Con esa lógica y respecto a los riesgos por POC's, puede decirse que su uso se justificó en aras a atender ciertos peligros -"hambruna", "sequía", "inundaciones" y/o "control de plagas"- toda vez que el desarrollo de la ciencia y la tecnología mostraban que eran convenientes (Aijón y Cumplido, 2007). Estudios posteriores mostraron que el uso de los POC's provocaba problemas más serios a mediano y largo plazo.

Extrapolando lo dicho en este apartado al caso de Sonora. Primero, los riesgos por POC's se asocian al uso de tales compuestos en ciertas prácticas y procesos agrícolas, quizá con el propósito original de evitar 
peligros como la sequía, plagas o hambruna; pero tales razones palidecen al observar los intereses de tipo económico involucrados, sobre todo aquéllos que tienen que ver con generar rendimientos crecientes que generasen mayores beneficios, ganancia o capital. Las novedosas prácticas agrícolas se consolidan y popularizan sin advertir sus efectos adversos en el medio ambiente y en la salud humana.

A la fecha, los riesgos por POC,s bien pueden sumarse a la lista de problemas ambientales complejos que están latentes en la llamada sociedad de riesgo y que poseen ese hilo conductor entre lo visible y lo invisible. Los POC's pueden estar presentes en todas partes, en suelos, agua, aire y alimentos (Sánchez 2001: 10-11) y expresarse en ciertas enfermedades en el largo plazo, excepto en intoxicaciones, riesgo que se torna mayor cuando se participa en determinados trabajos y estilos de vida.

El contexto en el cual se da la situación del riesgo y lo qué es posible hacer al respecto confirma que "... el trato con el medio y con la inseguridad se convierten biográfica y políticamente en una cualificación civilizatoria clave" (Beck, 1986: 85, en Ochoa Nogales, et al., 2012: 249).

$\mathrm{Al}$ igual que en otros problemas ambientales, la situación de riesgo que generan los POC's, reclama el compromiso y actuación de todo actor social posible -funcionarios públicos de instancias económicas, de salud y educativas, amas de casa, estudiantes, productores, etc.-. La demanda es de mayor responsabilidad social para avanzar hacia la conformación de eco-ciudadanos, esto es, personas conscientes de su responsabilidad social y ambiental a nivel local y planetario, que actúan en consecuencia. Los programas y estrategias de comunicación tienen ese gran reto: además de brindar conocimiento para la prevención del riesgo, motivar la reflexión y trabajar en la conformación de eco-ciudadanía que refuercen cambios de conducta en ese sentido. Sin embargo, en una problemática tan compleja afloran actores e instituciones con intereses y posibilidades de actuación bien demarcados.

Para afinar ideas y estar en posibilidades de delinear el contenido de una estrategia de comunicación, en el apartado siguiente se presentan algunas recomendaciones que organismos internacionales sugieren tomar en cuenta al diseñar un programa de comunicación.

\section{Lineamientos base de una Estrategia de Comunicación}

Se reconoce que es indispensable analizar el proceso de riesgo y se identifican tres aristas al proceder a tal análisis (ACS, 1998).

- La evaluación del riesgo para la salud o para los ecosistemas

- El manejo de ese riesgo

- La comunicación de ese riesgo 
Figura \#1. Proceso para el análisis de riesgos ambientales.

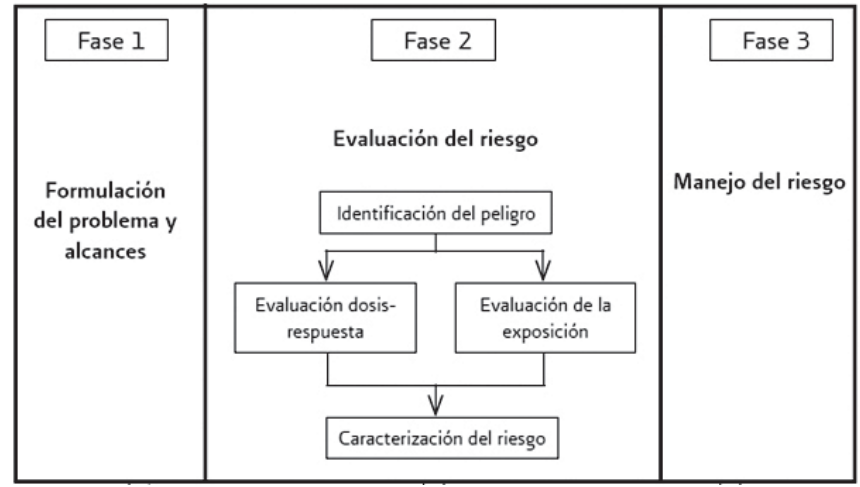

Fuente: Levy, 2009 y NAS, 1983 (en Ize, Zuk y Rojas-Bracho, 2010: 25).

Es importante señalar que el lugar que ocupa cada componente es indistinto, no representan una secuencia o un orden en el cual deban llevarse a cabo; la comunicación sin embargo desempeña un papel clave, de ahí necesidad de "...la participación formal de todos los afectados (stakeholders y la necesidad de ] ... incorporar la comunicación y la percepción del riesgo en todas las fases..." (Izze et al., 2010: 25).

La figura anterior muestra el procedimiento a seguir al atender riesgos ambientales y permite apreciar que la percepción y comunicación del riesgo son parte inherente de cada fase.

Para la Organización Panamericana de la Salud (OPS, 2003) un programa de comunicación contempla al menos ocho objetivos: 1) Promover el conocimiento y la comprensión del tema en todos los participantes, actores y sectores de interés; 2) Promover la consistencia y la transparencia sobre la toma de decisiones y la instrumentación de medidas de manejo del riesgo; 3) Proveer una base sólida para entender las decisiones de manejo del riesgo propuestas o implementadas; 4) Mejorar la eficacia y la eficiencia del proceso de análisis del riesgo; 5) Contribuir al desarrollo y la entrega de información y programas de educación efectivos; 6) Promover la confianza pública en las instituciones encargadas de tomar decisiones; 7) Promover la participación de todos los sectores interesados; 8) Intercambiar información sobre actitudes, conocimientos, valores, prácticas y percepciones relativas a los riesgos.

Establecidos los objetivos de la estrategia de comunicación, se definen sus líneas de acción. Ambos deberán ser coherentes y apuntar a eliminar, reducir o mitigar el problema de interés, en este caso, los riesgos por POC's. Los ejes o líneas de acción son las estructuras de referencia operativa 
que permitirán tomar decisiones sobre los procedimientos a realizar para lograr los procesos participativos de la comunidad en el campo de acción (cfr. OPS, 2003).

Para contextualizar tal proceso de comunicación y guiar su respectiva planeación, hay que responder tres preguntas importantes: el ¿quién?, el ¿qué? y el ¿cómo? de la estrategia (INE-SEMARNAP, 2000: 37).

El "quién" remite a diferenciar los dos tipos de actores que constituyen y dan forma-sentido al proceso de comunicación: quién comunica y quién recibe la comunicación. Se establece el perfil de cada grupo, los criterios que deben cumplir las personas que participan como receptores del proceso de comunicación (niños, estudiantes de preparatoria, jornaleros/as agrícolas, amas de casa, etc.) y los requisitos que deben cumplir quienes desempeñarán el papel de comunicador.

Para diseñar la estrategia se sugiere partir de la información que, sobre el tema de interés, posee el grupo receptor de la misma. Se sugiere acercarse a un grupo representativo de éste o que al menos cumpla el perfil de tal población objetivo, para indagar por el conocimiento que tiene sobre el tema en cuestión. El propósito es identificar los aspectos de la comunidad receptora que reflejan desconocimiento, poca información, prácticas equivocadas o deficientes respecto al tema central del mensaje a comunicar. En base a tal información se ajusta la estrategia de comunicación, se matiza o enfatiza su contenido.

El "qué" es la otra pregunta que orienta el diseño de la estrategia de comunicación y es la que refiere al contenido del mensaje. Se procede entonces a definir el contenido del mensaje a comunicar. Deberán utilizarse conceptos claros, básicos, sencillos. El mensaje deberá destacar el problema existente para motivar a la población a conocer las condicionantes del riesgo así como las medidas a seguir para su prevención. Se trata de dejar claro un pensamiento importante: "más vale prevenir que remediar". El mensaje tiene el fin de concientizar, informar y propiciar un cambio de conducta que disminuya el riesgo.

Finalmente el "Cómo" indica la transmisión del mensaje, la forma en la que éste será transmitido. Para avanzar en tal propósito, es conveniente recordar los cuatro objetivos que destacan en el proceso de comunicación de riesgos: a) aumentar la conciencia sobre el riesgo; b) informar sobre el riesgo; c) construir un consenso entre la población y la institución que evalúa o maneja el riesgo; y d) cambiar la conducta ante el riesgo. En esa perspectiva, el Instituto Nacional de Ecología sugiere combinar mensajes escritos, orales y visuales, así como diversas aplicaciones por computadora y la interacción con la población (INESEMARNAP, 2000: 81-85). A continuación, una precisión y algunas recomendaciones en cada caso. 
Los mensajes escritos se utilizan en circulares, volantes, trípticos, folletos e historietas. Pueden ser ilustraciones u otros elementos gráficos que difieren de las presentaciones visuales porque sus mensajes son más largos y complejos. Para elaborar los mensajes escritos se sugiere considerar: a) la naturaleza del riesgo; b) las alternativas de acción requeridas y los riesgos asociados a esas alternativas; c) la incertidumbre en la evaluación de riesgos; d) el cómo se manejará el riesgo; e) los beneficios del riesgo; f) el punto de contacto para adquirir información al respecto; g) las acciones recomendables para mitigar o manejar la exposición al riesgo; h) las metas y contenido del mensaje escrito; i) otra información proveniente de medios diversos (glosario, sugerencias, tablas, lista de información adicional); j) datos de la institución responsable de la comunicación (teléfonos, dirección, etc); k) directorio de otras instituciones y personas que pueden asesorar/ auxiliar.

Los mensajes orales refieren a cuando alguien habla directamente a una persona o población, sobre todo en los casos que no hay mucha oportunidad de interactuar (presentaciones en escuelas, asociaciones, centros de salud, etc.). La recomendación es: a) preparar y dar a la población algo que le ayude a recordar; b) elegir un lugar apropiado, neutral; c) cuidar que la acústica y la iluminación sean apropiadas; d) usar la ropa adecuada para el lugar y la ocasión; e) reforzar el mensaje con apoyo visual atractivo y legible; f) hablar el idioma de la población (lengua materna y lenguaje coloquial); g) no prometer lo que no se puede cumplir; h) cumplir lo que se prometió; i) elegir un vocero formal, creíble, aceptado por la población e institución, que sea buen orador, responda bien y sea consciente de las reglas y políticas del trabajo de la institución respecto al problema. Se sugiere que participe un solo exponente por sesión -las presentaciones institucionales numerosas pueden intimidar-; tal ponente debe ser la persona apropiada dentro y fuera de la institución de interés; anticipar las respuestas apropiadas a las preguntas posibles así como éstas últimas; seguir todas las recomendaciones respecto al lenguaje descritas en la sección de mensajes escritos.

Los mensajes visuales son los que contienen elementos gráficos y relativamente poco texto. Generalmente están plasmados en carteles, trípticos, posters, videos, anuncios e incluso videoconferencias. El INESEMARNAP (2000: 84-85) recomienda: a) utilizar en el texto un lenguaje apropiado a la población objetivo -nivel escolar y comprensión de lectura-; b) centrar el mensaje en sus preocupaciones y necesidades de información; c) utilizar un mensaje limitado, simple, claro y de lectura rápida; d) los elementos gráficos deben reforzar el texto sobre el mensaje que se desea transmitir, no contradecirlo ni confundirlo; e) colocar los mensajes en sitios visibles para la población objetivo; f) incluir fuentes de información adicional de las personas o instituciones que lo promueven (teléfonos o direcciones para contacto futuro).

Todos estos son los referentes que se sugiere utilizar como una guía al diseñar una estrategia de comunicación. En el caso que ocupa 
esta investigación, elegida la población objetivo, se ajusta el mensaje a comunicar a las necesidades de información que tal población exhibe sobre los riesgos por POC's.

\section{Percepción del riesgo por POC's en Jornaleros/as Agrícolas de Pesqueira, Sonora, México}

En el estado de Sonora, todos los jornaleros/as agrícolas que viven o trabajan cerca del ambiente donde se aplican productos químicos para el control de plagas -hubiesen o no trabajado en la aplicación directa de plaguicidas-, están en situación de riesgo por POC's aún sin estar en aparente exposición (Silveira, et al., 2011: 71). Es el caso de los jornaleros/as y sus familias-, que laboran y/o viven en o cerca de localidades agrícolas ubicadas en los Valle del Yaqui (Cajeme, Navojoa, Empalme), San Luis Río Colorado y/o la zona centro (Costa y Pesqueira). Se decide que los receptores de la estrategia de comunicación serán los jornaleros/as agrícolas que residen en la localidad de Pesqueira, Sonora. Se aplica una encuesta de opinión sobre riesgos por POC's a una muestra representativa de dicha población que fue elegida de manera aleatoria simple utilizando la fórmula siguiente:

Donde,

$n=\underline{K^{2} P Q N}$

$\mathrm{e}^{2}\left(\overline{\mathrm{N}-1)+\mathrm{K}^{2}} \mathrm{PQ}\right.$

$\mathrm{n}=$ muestra simple

$\mathrm{N}=$ tamaño total de la población

$P=0,5$ para un tamaño máximo de la muestra

$Q=0,5$

$\mathrm{e}^{2}=$ error muestral que se ha fijado en 0.055

$\mathrm{K}=$ contante que depende de la confianza deseada $=2$ para un nivel de confianza del $95.5 \%$.

Tal estimación resulta en 289 jornaleros/as. Se les aplica un cuestionario constituido por 108 reactivos agrupados en tres bloques: 1) información de tipo sociodemográfico; 2) información sobre conocimiento de los riesgos que generan los POC's en la salud y en el medio ambiente; 3 ) prácticas de cuidado personal e higiene personal que llevan a cabo en su vida cotidiana (hogar/trabajo). A continuación, los principales resultados. 


\section{Cuadro 1. Jornaleros/as Agrícolas de Pesqueira, Sonora. Datos sociodemográficos}

\begin{tabular}{|c|c|c|c|}
\hline \multirow{2}{*}{ Varlatie } & \multirow{2}{*}{ Atributo: } & \multicolumn{2}{|c|}{ focusncla } \\
\hline & & $\mathbf{n}$ & $\%$ \\
\hline \multirow[t]{3}{*}{$50 \times 0$} & D.TL: & 109 & 37.7 \\
\hline & Hongre......... & 154 & 53.3 \\
\hline & Wo conie: is & 26 & 90 \\
\hline \multirow[t]{4}{*}{ Bad } & 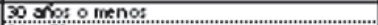 & 185 & $6+9$ \\
\hline & 31050 & 54 & 18.7 \\
\hline & 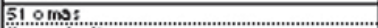 & ...t. & .8 .3 \\
\hline & Wo conk: is & 26 & 90 \\
\hline \multirow[t]{4}{*}{ Ectado CIvI } & 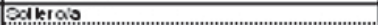 & 157 & 54.3 \\
\hline & casodourionitibe & 96 & 332 \\
\hline & 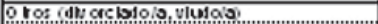 & 10 & 3.5 \\
\hline & Wo conk: is & 26 & 90 \\
\hline \multirow[t]{5}{*}{ tuoside kalm lento } & 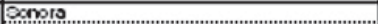 & $1+3$ & 49.5 \\
\hline & cento deipar & 82 & 30.4 \\
\hline & 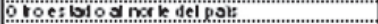 & 32 & iii \\
\hline & bar dei pas & 6 & 2.1 \\
\hline & Mo conks bs & 26 & 90 \\
\hline \multirow[t]{3}{*}{ ahocde viur on sonera } & 13 anos o mst & 187 & 64.7 \\
\hline & 12 oponomos & 64 & 33 \\
\hline & Ho conk: is & 38 & 13.1 \\
\hline \multirow[t]{4}{*}{ TIpo de Re cldenola on senera } & 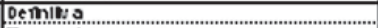 & 223 & 77.2 \\
\hline & Tempora & 25 & 8.7 \\
\hline & Wo sabe & 9 & 3.1 \\
\hline & Moconks is. & 32 & 11.1 \\
\hline \multirow{3}{*}{ Origen Indioena } & ة & 66 & 238 \\
\hline & Mo & 197. & 682 \\
\hline & Ho canie:io & 26 & 90 \\
\hline \multirow{3}{*}{ Grupo Indioena de per te ne nola } & zas.op: & 29 & 11.1 \\
\hline & "Triqu & $2 i$ & 80 \\
\hline & 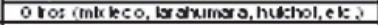 & 16 & 6.1 \\
\hline \multirow{3}{*}{$\begin{array}{l}\text { Mam ere de percenac que } \\
\text { In te oran cu fam lila }\end{array}$} & 5 o me mot & 113 & 430 \\
\hline & De 6 onst.... & $1+8$ & $5 \%$ \\
\hline & no conk: io & 28 & 9.7 \\
\hline \multirow{4}{*}{ Mam ere de hlle cque tiene } & 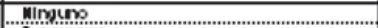 & 163. & 5.t. \\
\hline & 3 o me no: & 44 & 152 \\
\hline & t.oms & 56 & 19.4 \\
\hline & No conie:io & 26 & 90 \\
\hline \multirow{3}{*}{ VIvo oon eu fam Illa } & 9! & $2+8$ & 943 \\
\hline & Mo & 15 & 5.7 \\
\hline & Mo conk:!o & 26 & 90 \\
\hline \multirow{4}{*}{ Adultoc vivendoen oa ca } & 102 & 100 & 380 \\
\hline & $30 \mathrm{~ms}:$ & 153 & 58 \\
\hline & Mo conk:10 & 36 & 12.5 \\
\hline & Tolv & 289 & 100.0 \\
\hline \multirow{4}{*}{ minoc unendo en oaca } & 102 & 104 & 39.5 \\
\hline & 3o ons: & iio & 449 \\
\hline & Ho conk:io & 67 & 232 \\
\hline & Tögi........... & 283 & 1000 \\
\hline \multirow{5}{*}{ Eduosolon form al } & Mo kne & 13 & 4.9 \\
\hline & 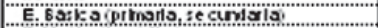 & 101 & 3.4 \\
\hline & E.ikdis (bociliki) & $1+7$ & 55.9 \\
\hline & Espeivaliseroira & 2 & 08 \\
\hline & no conk: io & 26 & 90 \\
\hline \multirow{4}{*}{$\begin{array}{l}\text { Alfab e tisalon cabe loery } \\
\text { e cerlbir) }\end{array}$} & 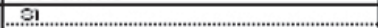 & $2 ! 3$ & 810 \\
\hline & $P \circ \circ$ & 27 & 10.3 \\
\hline & Ho & 23 & 8.7 \\
\hline & Mo conk: 10 & 26 & 90 \\
\hline PobI & a olo n Total & 289 & 100.0 \\
\hline
\end{tabular}

Fuente: Resultados de encuesta aplicada a jomalerosias agńcolas residentes de Pesqueira,

Sonora, México de octubre a diciembre del 2011.

\section{Información de tipo sociodemográfica de Jornaleros/as (Cuadro \#1)}

De los 289 participantes en el estudio, 53\% son hombres y 38\% mujeres, el 9\% restante no contestó. Su edad fluctúa de los 15 a los 64 años de edad pero predomina el grupo de 15 a 30 años (64\%), seguido por el de 
adultos de 31 a 50 años (18\%). El grupo de solteros supera a los que viven en pareja, $54 \%$ vs $33 \%$.

En cuanto a lugar de nacimiento, cerca de la mitad anotó Sonora (49\%), el 28\% el centro del país y el 11\% algún otro estados del norte. De origen indígena resultó ser el $23 \%$, y de estos, la mayoría son zapotecos o triquis. En cuanto a tiempo de residir en Sonora, el 65\% anotó 13 años o más, el $22 \%$ doce años o menos, el 13\% restante no contestó. El $77 \%$ vive de manera definitiva en Pesqueira, el 9\% de manera temporal, el 3\% marcó "no sé" y el resto no contestó.

En datos familiares, la mayoría anoto que vive con su familia (94\%) la cual se integra por seis o más miembros en el mayor de los casos $(56 \%)$. El 57\% de los informantes no tiene hijos y el 19\% tiene cuatro o más. En la mayoría de las casas/habitación viven más de tres adultos y también más de tres niños.

En escolaridad, el 38\% de los participantes en la encuesta cuenta con estudios de nivel básico, el 56\% con estudios de bachiller y el $14 \%$ ninguno o no contestaron. El 81\% marcó que sí saben leer, el 10\% que poco, el $8.7 \%$ que no sabe y el $9 \%$ no respondió.

\section{Tabla 2. Jornaleros/as Agrícolas de Pesqueira, Sonora. Ingreso y gasto familiar}

\begin{tabular}{|c|c|c|c|}
\hline \multirow{2}{*}{ Re activo } & \multirow{2}{*}{ Opciones de Respuesta } & \multicolumn{2}{|c|}{ si } \\
\hline & & $\mathrm{N}$ & $\%$ \\
\hline \multirow{3}{*}{ Personas que con tribuyen al gasto familiar } & 102 & 173 & 60 \\
\hline & 304 & 96 & 33 \\
\hline & 50 más & 19 & 7 \\
\hline \multirow{3}{*}{ Ingreso Semanal } & $\$ 848$ o menos $(<2 \mathrm{sms})^{*}$ & 167 & 58 \\
\hline & $\$ 849$ a $\$ 1284(>2 s m s,<3 s m s)^{*}$ & 85 & 29 \\
\hline & $\$ 12850$ más $(>3 s \mathrm{~ms})^{*}$ & 17 & 6 \\
\hline \multirow{4}{*}{ Ingreso Familiar Acumulado Semanal } & $\$ 848$ o menos $(<2 \mathrm{sms})^{*}$ & 68 & 24 \\
\hline & $\$ 849$ a $\$ 1284(>2 s m s,<3 s m s)^{*}$ & 71 & 25 \\
\hline & $\$ 1285$ a $\$ 2543(>3 s m s,<5 s m s)^{*}$ & 106 & 37 \\
\hline & $\$ 2544$ o más $(>5 \mathrm{sms})^{*}$ & 24 & 8 \\
\hline \multirow{2}{*}{$\begin{array}{l}\text { El Ingreso familiar acumulado es suficiente } p \text { ara } \\
\text { cubrir el gasto familiar }\end{array}$} & sí & 91 & 31 \\
\hline & No & 193 & 67 \\
\hline
\end{tabular}

Fuente: Resultados de encuesta aplicada a jomaleros/as agrícolas residentes de Pesqueira, Sonora, México de octubre a diciembre del 2011

En cuanto a ingresos y gasto familiar (tabla \#2), la mayoría (60\%) anotó que sólo una o dos personas contribuyen al gasto familiar. En el 58\%, el ingreso semanal es menor a dos salarios mínimos; al observar el ingreso 
familiar acumulado llama la atención que en el $24 \%$ de los casos se mantiene ese mismo ingreso, en el $25 \%$ se ubica entre dos y tres, en el $37 \%$ entre tres y cinco, y sólo en el $8 \%$ de los casos tal ingreso es superior a los cinco salarios mínimos. No obstante, la mayoría (67\%) anotó que el ingreso semanal acumulado es insuficiente para cubrir los gastos familiares.

Al preguntarles por la edad que tenían cuando empezaron a trabajar en el campo (tabla \#3), la mayoría ( $80 \%$ ) anotó que 17 años o menos; generalmente lo han hecho en el sector agrícola $(75 \%)$; regularmente seis o siete días a la semana (55\%); ocho o más horas a la semana (63\%). Predomina pues, una jornada de trabajo de ocho o más horas diarias durante los seis o siete días que trabajan por semana.

Tabla 3. Jornaleros/as Agrícolas de Pesqueira, Sonora. Edad inicial de trabajo, sector de ocupación y jornada laboral (días/horas)

\begin{tabular}{|c|c|c|c|}
\hline \multirow{2}{*}{ Variable } & \multirow{2}{*}{ Atributos } & \multicolumn{2}{|c|}{ Frecuencia } \\
\hline & & $\mathbf{n}$ & $\%$ \\
\hline \multirow{3}{*}{$\begin{array}{l}\text { Edad inicial de } \\
\text { trabajo }\end{array}$} & 17 años o menos & 228 & 79 \\
\hline & 18 ańos o más & 20 & 7 \\
\hline & Sin respuesta & 41 & 14 \\
\hline \multirow{4}{*}{$\begin{array}{l}\text { Sector económico en } \\
\text { el que ha trabajado } \\
\text { con más frecuencia }\end{array}$} & Agricultura & 218 & 75 \\
\hline & Comercio & 6 & 2 \\
\hline & Otros (oficios, hogar, ganaderia) & 11 & 4 \\
\hline & Sin respuesta & 54 & 19 \\
\hline \multirow{4}{*}{$\begin{array}{l}\text { Días que trabaja por } \\
\text { semana }\end{array}$} & 50 menos & 57 & 20 \\
\hline & 607 & 159 & 55 \\
\hline & Solo en vacaciones & 6 & 2 \\
\hline & Sin respuesta & 67 & 23 \\
\hline \multirow{3}{*}{$\begin{array}{l}\text { Horas diarias de } \\
\text { trabajo }\end{array}$} & 7 horas o menos & 29 & 10 \\
\hline & 8 horas o más & 181 & 63 \\
\hline & Sin respuesta & 79 & 27 \\
\hline
\end{tabular}

Fuente: Resultados de encuesta aplicada a jomaleros/as agricolas residentes de Pesqueira, Sonora, México en octubre-diciembre del 2011.

\section{Vestuario y Posibles Prácticas de Riesgo en el Trabajo y en el hogar}

A pregunta expresa " ¿conocen el efecto que tienen los POC's en su salud y en el medio ambiente?", la mayoría de los jornaleros/as contestó que sí (85\%). Sin embargo, las respuestas brindadas a otros reactivos en relación a las prácticas de higiene y cuidado personal en su hogar y trabajo (campo agrícola), llevan a suponer que tal conocimiento es muy limitado (tabla 4). 
Respecto al vestuario que utilizan de manera regular al trabajar en los campos agrícolas, la mayoría anotó que no usa mandil $(92 \%)$, ni ropa resistente a químicos $(75 \%)$, ni botas $(68 \%)$, ni pañuelos $(61 \%)$, ni mascarillas $(56 \%)$ ni lentes $(55 \%)$. Algunos tampoco utilizan guantes $(32 \%)$, pantalón grueso $(23 \%)$ o camisa manga larga $(22 \%)$. Por tanto, se concluye que es muy limitado el vestuario y los implementos de protección que usan la mayoría de los jornaleros/as. Habrá que indagar si esto obedece a que carecen de esa ropa e implementos o si es por descuido u olvido. De ser esto último, falta también averiguar si ese vestuario "disponible" es facilitado por el empleador, si está en el campo o se lo lleva a su casa.

\section{Tabla 4. Conocimiento y Prácticas de Seguridad en el trabajo y hogar.}

\begin{tabular}{|c|c|c|c|c|c|}
\hline & \multirow{3}{*}{ Yariablefitem } & \multicolumn{4}{|c|}{ Fra $\alpha$ ancia $289=100 \%$} \\
\hline & & \multicolumn{2}{|c|}{ NO } & \multicolumn{2}{|c|}{ SI } \\
\hline & & $n$ & $\%$ & $\mathbf{n}$ & $\%$ \\
\hline Se que son les COP y algunos des: & us efectos en la salud amblemtaly humana. & 44 & 150 & 245 & 85.0 \\
\hline \multirow{9}{*}{$\begin{array}{l}\text { Vestuario que usa regularmente } \\
\text { en su trabajo, en el campo } \\
\text { agricola }\end{array}$} & Camisa manga larga. & 65 & 225 & 224 & 85.2 \\
\hline & Pantalon Grueso. & 67 & 23.2 & 222 & 84.4 \\
\hline & 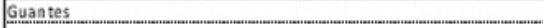 & 92 & 3.18 & 197 & 74.9 \\
\hline & 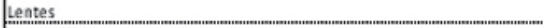 & 145 & 55.1 & 144 & 49.8 \\
\hline & Mascarillas & 148 & 56.3 & 141 & 48.8 \\
\hline & Pa huelos & 161 & 612 & 128 & 44.3 \\
\hline & Botas & 178 & 67.7 & 111 & 38.4 \\
\hline & Ropa res istente a qu imicos & 198 & 753 & 91 & 31.5 \\
\hline & Ma ndil & 242 & 920 & 47 & 16.3 \\
\hline \multirow{7}{*}{$\begin{array}{l}\text { Precauciones que toma antes y/o } \\
\text { después de su trabajo en el } \\
\text { campo }\end{array}$} & Lavarse con agua y la bon & 224 & 275 & 65 & 24.7 \\
\hline & Tra bajar con cuidado/p eca udión alejado de las zonas con pla guicidas & 237 & 820 & 52 & 19.8 \\
\hline & Utilizar equ ipo de protección (mascarilla, gua ntes etc) & 268 & 927 & 21 & 8.0 \\
\hline & Bañarse & 274 & 948 & 15 & 5.7 \\
\hline & Abs tegerse de comero fumarcuando están trabajando en el cameo & 276 & 95. & 13 & 4.9 \\
\hline & Usar imple mentos de protección en su cuerpo y rostro & 278 & 962 & 11 & 4.2 \\
\hline & Dejarde in gerir bebidas alcohólicas & 284 & 983 & 3 & 1.9 \\
\hline \multirow{7}{*}{ Prácticas de riesgoen el trabajo } & Conoce alguna resoludón que señala a qué distancia debe fu migar... & 253 & 87.5 & 36 & 12.5 \\
\hline & Ha sentido algu na vez la fuga de sustandas toxicas. & 246 & 85.0 & 43 & 14.9 \\
\hline & Nunca ha es tado cerca de donde se aplican plaguicidas. & 200 & 69.0 & 89 & 31.0 \\
\hline & Ha a plica do pla guicidas & 147 & 310 & 142 & 49.0 \\
\hline & Lava s us manos antes de ir al baño. & 39 & 135 & 250 & 85.5 \\
\hline & Me lavo las ma nos antes de consumiralime ntos. & 39 & 40 & 260 & 96.0 \\
\hline & Me lavo las ma nos cuando consumo productos del campo & 171 & 93.0 & 18 & 6.0 \\
\hline \multirow{6}{*}{$\begin{array}{l}\text { Destinofinal de productos de } \\
\text { riesgofdepósitos, residuos, ropa } \\
\text { usada al aplicar plaguididas\} }\end{array}$} & Un centro de a co pio (se recicla n o peutiliza n) & 271 & 93.8 & 18 & 6.2 \\
\hline & Depósito o contened or es pecial & 278 & 96.2 & 11 & 3.8 \\
\hline & Se almace nan en el campo & 280 & 969 & 9 & 3.1 \\
\hline & 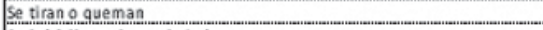 & 281 & 972 & 8 & 28 \\
\hline & Se lo(s) llevan los trabajadores. & 287 & 993 & 2 & 0.7 \\
\hline & Nosé & 74 & 25.6 & 215 & 74.4 \\
\hline \multirow{2}{*}{ Prácicas de riesgoen ell hogar } & Almacenar agua en ba ldes o depósitos que contuvieron plaguididas & 232 & 800 & 57 & 19.7 \\
\hline & Nimacenar algún depósito con plaguicida ea su casa & 264 & 910 & 25 & 8.7 \\
\hline \multicolumn{6}{|c|}{$\begin{array}{l}\text { Fuente: Resultados de encues ta aplicada a jornalerosias agrícol as res identes de Pesqueira, So nora, Mexico de octubre diciembre } \\
\qquad \mathbf{2 0 1 1 .}\end{array}$} \\
\hline
\end{tabular}

$\mathrm{Al}$ indagar por los hábitos de higiene y prácticas de cuidado o de tipo preventivo que deberían tomar en su trabajo -antes y después de la situación de riesgo a la que han estado expuestos-, la información resultante se torna alarmante. La mayoría de los trabajadores agrícolas olvidan lavarse las manos con agua y jabón (77\%); olvidan tener cuidado/precau- 
ción y alejarse de las zonas de aplicación de plaguicidas (82\%); no utilizan equipo de protección en el cuerpo ni en el rostro (93\%); comen y fuman en el campo (95\%). El 96\% no se protegen el cuerpo ni el rostro. Sólo el 2\% se abstiene de tomar bebidas alcohólicas en el trabajo y apenas el 5\% anotó que se bañan inmediatamente al llegar a sus casas.

\section{Tabla 5. Jornaleros/as Agrícolas de Pesqueira. Padecimientos o enfer- medades regulares o frecuentes.}

\begin{tabular}{|c|c|c|c|}
\hline \multirow{3}{*}{\multicolumn{2}{|c|}{$\begin{array}{l}\text { Enfermedades o malestares que sufre él o algun miem bro } \\
\text { de su familia }\end{array}$}} & \multirow{2}{*}{\multicolumn{2}{|c|}{$\begin{array}{l}289=100 \% \\
\text { SI }\end{array}$}} \\
\hline & & & \\
\hline & & $\mathbf{n}$ & $\%$ \\
\hline \multirow{8}{*}{ 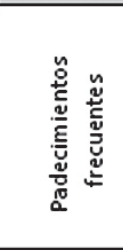 } & Dolor de cabeza & 150 & 52 \\
\hline & Irritación de la piel & 98 & 34 \\
\hline & Mareos & 78 & 27 \\
\hline & Vómitos & 61 & 21 \\
\hline & Náuseas & 58 & 20 \\
\hline & Disrupción cutánea... & 50 & 17 \\
\hline & Visión borrosa & 47 & 16 \\
\hline & No sabe & 40 & 14 \\
\hline \multirow{10}{*}{ 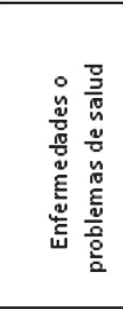 } & & 169 & 59 \\
\hline & Diabetes & 69 & 24 \\
\hline & Asma & 37 & 13 \\
\hline & Problemas de aprendizaje & 32 & 11 \\
\hline & Artritis reumatoide & 30 & 10 \\
\hline & Problemas cancerígenos & 29 & 10 \\
\hline & Enfermedades no especificadas & 20 & 7 \\
\hline & Daños o de sórdenes reproductivos & 18 & 6 \\
\hline & Hipersensibilidad & 13 & 5 \\
\hline & Otros $(*)$ & 12 & 4 \\
\hline \multicolumn{4}{|c|}{$\begin{array}{l}\text { () Cada uno de los padecimientos y enfermedadas siguientes fuer on nombrados } \\
\text { por } 4 \% \text { o menos de los jornalerosias partic ipantes en el estudio: perturbaciones al } \\
\text { sistema inmunológico, daño al sistema nervioso centraly periféric } 0 \text {, insensibilidad, } \\
\text { daño neurotóxico, alteraciones al sistema hormonal, def ectos de nacimiento, } \\
\text { poblemas en la sangre (plaquetas, leucemia, anemia, etc.), presión, daño al ADNy } \\
\text { apoptosis. }\end{array}$} \\
\hline
\end{tabular}

Fuente: Encuesta aplicada a jornaleros/as agrícolas residentes de

Pesqueira, Sonora, México entre octubrey dici embre del 2011.

Para complicar más la situación, hay otras prácticas de riesgo en su trabajo. El $87 \%$ de los trabajadores agrícolas desconoce a qué distancia deben ser aplicados los fumigantes; el 69\% reconoce haber estado cerca de donde se aplican plaguicidas y el $49 \%$ anotó que los ha aplicado; y un 15\% expresó que sí ha sentido alguna vez la fuga de sustancias tóxicas. Afortunadamente la mayoría se lavan las manos antes de ir al baño (86\%) y de consumir alimentos (96\%). Sin embargo, el 93\% no lo hace cuando consume productos hortofrutícolas del mismo campo (uvas, calabazas, melón, zanahorias y/o sandía). 
Al preguntarles si conocen el destino final de ciertos productos de riesgo (depósitos y residuos de productos contaminantes o ropa usada al aplicar plaguicidas), el 4\% señaló que se llevan a depósito o contenedor especial, el 3\% anotó que se almacenan en el propio campo o bien que se tiran o queman. Llama la atención que el 74\% anotó no saber el destino de tales productos contaminantes. Sin embargo, al indagar por las prácticas de riesgo en su hogar, llama la atención que el $20 \%$ de los trabajadores agrícolas reconocen que utilizan los depósitos contenedores de plaguicidas para almacenar agua e incluso un $9 \%$ guarda en su casa algún depósito con plaguicida. Esta práctica es sumamente riesgosa.

\section{Conocimiento de riesgo por POC's en la salud y acciones preventivas}

En este aspecto se indaga por las enfermedades/malestares que ha(n) padecido él o algún miembro de su familia; por las actividades que suponen riesgos a la salud; por las enfermedades/malestares que a su juicio derivan del trabajo que realiza como jornalero/a; y los efectos que la aplicación de plaguicidas tiene en la salud; así como por las vías de acceso de los plaguicidas al cuerpo humano.

En cuanto a los malestares o enfermedades padecidas por él o alguien de su familia (tabla 5), destacan "dolor de cabeza" (52\%), "irritación de la piel" (34\%), "mareos" (27\%), "vómitos" (21\%) y "nauseas" (20\%) en el caso de malestares; y en enfermedades, "alergias" (59\%), "diabetes" (24\%) y “asma” (13\%).

Al pedirles que anotaran las actividades que realizan como trabajadores agrícolas y que consideran son un riesgo para la salud, el 51\% marcó "ninguna" y sólo el 16\% anotó "las actividades que realiza en el campo". El $24 \%$ anotó "aplicación de plaguicidas".

El 53\% de los participantes en el estudio no vincula sus problemas de salud con el trabajo que realizan en el campo. No obstante, al $47 \%$ restante está preocupado por tal hecho. A la cuarta parte, por ejemplo, le preocupa "padecer alguna enfermedad relacionada con los plaguicidas" y apenas al $4 \%$ “...el uso de químicos". Esto es paradójico ya que buena parte de esta población ha señalado que al trabajar en el campo sufre "dolor de cabeza", "fatiga" "mareos", "nauseas, "vómitos", "somnolencia" y/o "falta de respiración". Incluso el 18\% atribuyó tales malestares al uso/efecto de aplicar plaguicidas y el 14\% a una deshidratación por calor, 27 personas expresaron que han sufrido intoxicación.

De presentarse un problema de salud en el centro de trabajo, la mayoría ( $80 \%)$ anotó que acudiría al centro de salud más cercano y realizarían tres acciones: "tomar leche" (38\%), "lavarse las manos" (33\%) y "cambiarse de ropa" $(17 \%)$. 
Y respecto a las vías de acceso de los plaguicidas al cuerpo humano reconocidas por este grupo de jornaleros/as, la más citada fue la "respiratoria" (70\%), seguida por "cutánea" (55\%), "boca" (49\%) y también marcaron "por los ojos" $(40 \%)$-la sumatoria de los porcentajes es más de 100\% porque marcaron más de una opción-.

Con esta información de partida, se procede a bosquejar el contenido de la estrategia de comunicación.

\section{Contenido de estrategia de comunicación... tareas pendientes}

En seguimiento a directrices marcadas en apartado anterior, fueron acotados el propósito, las líneas de acción y el mensaje a comunicar. Se confirma que el propósito central de la estrategia de comunicación será facilitar información a los jornaleros/as agrícolas de Pesqueira, Sonora para orientarlos a realizar ciertas acciones en su vida cotidiana (hogar y trabajo) que les permita mitigar la situación de riesgo por POC's en la que se encuentran. Los objetivos que sustentan y orientan el proceso de comunicación de riesgos en este caso son: a) aumentar la conciencia sobre el riesgo; b) informar sobre el riesgo; c) cambiar la conducta ante el riesgo. Hoy por hoy, queda fuera de la estrategia aquél otro que refería a "...construir un consenso entre la población y la institución que evalúa o maneja el riesgo...". No se ha considerado en esta ocasión realizar una evaluación pre-post de la estrategia de comunicación.

Del instrumento aplicado para conocer la opinión que los jornaleros/as tienen respecto a los POC's y riesgos implicados, se concluye que efectivamente existen varias prácticas de riesgo, algunas referentes al vestuario utilizado y otras a ciertas acciones de cuidado e higiene personal en su vida cotidiana (hogar y trabajo). Por tanto, la información que integra el programa de comunicación se organiza en torno a tres líneas de acción: 1) los POC's y riesgos asociados en la salud ambiental y humana; 2) Prácticas de cuidado e higiene personal para la prevención del riesgo por POC's en el trabajo (campo); 3) Prácticas de cuidado e higiene personal para la prevención del riesgo por POC's en el hogar.

Las líneas de acción confluyen y son congruentes con el propósito general de prevenir los riesgos por POC's en la salud de los jornaleros/as agrícolas y de sus familias. Cada eje o línea de acción constituye las estructuras de referencia operativa que guiará la toma de decisiones en torno a los procedimientos a realizar en el campo a fin de lograr los procesos participativos de interés. Aspecto que resulta de gran importancia al concretar la estrategia en el área geográfica y población de interés, sobre todo cuando el grupo receptor se amplía a los familiares de los jornaleros/as agrícolas, a sus parejas e hijos/as. 
El otro actor o personaje clave de la estrategia de comunicación es el grupo emisor del mensaje. Este personaje está casi siempre conformado por comunicadores o, como ha sucedido en este proyecto, por personal competente que ha participado en la investigación-diagnóstico y que ha mostrado: a) conocimiento de la literatura en torno a la comunicación de riesgos por POC's, percepción del riesgo y población objetivo; b) experiencia en aplicación e interpretación de encuestas, con preferencia si participaron en la de este proyecto; c) interés, habilidades y aptitudes en el campo de la comunicación. En relación al perfil y cualidades de este personaje, es importante señalar también que "...escuchar es fundamental en el proceso de comunicación de riesgos para la salud pública y puede ayudar a las autoridades de salud pública a entender lo que la gente piensa, las necesidades de información comunitaria y las fuentes de información confiables en una región afectada" (OMS, 2008: 17).

Respecto al mensaje a comunicar propiamente dicho, estos se ha ido afinando (matizando o enfatizando) según sean las necesidades de información que presenta la población receptora en lo que concierne a cada referente o línea de acción. El cuestionario de opinión aplicado a los jornaleros/as con el fin de hacer tal diagnóstico permitió identificar varias acciones que llevan a cabo en su vida cotidiana (hogar y trabajo) que agravan su situación de riesgo. Se detectó que predominan acciones erróneas e inadecuadas tanto en el campo como el hogar las cuales se atribuyen a desconocimiento, desinterés o descuido.

Así, por ejemplo, en relación al vestuario (pantalón grueso, camisa manga larga, zapatos especiales, entre otros) y equipo de protección (cubre-bocas, mandil, mascarilla, guantes, etc.) que utiliza la población al trabajar en el campo, se aprecia que no es el adecuado para evitar precisamente el riesgo por exposición a contaminantes. Por tanto, el mensaje para orientar un cambio de conducta al respecto puede ser:

“...Utilizar vestimenta adecuada en el campo disminuye el riesgo de que sustancias tóxicas entren al cuerpo humano..." o "...Utilizar el equipo de protección al aplicar químicos disminuye el riesgo de que sustancias tóxicas entren al cuerpo humano por los ojos, la boca, la nariz o la piel..." o enunciados juntos el mensaje sería: "Utilizar vestimenta adecuada en el campo y un equipo de protección al aplicar químicos disminuye el riesgo de que sustancias tóxicas entren al cuerpo humano por los ojos, la boca, la nariz o la piel”.

Otra práctica riesgosa identificada fue el uso de envases contenedores de plaguicidas como depósitos de agua. Lo cual justifica integrar al programa de comunicación un mensaje orientado a cambiar tal práctica:

“...Almacenar envases de plaguicidas en la vivienda está asociado con problemas en la salud. NO hacerlo disminuye el riesgo de sufrir malestares y enfermedades..." 
Los tres mensajes enunciados -utilizar vestimenta adecuada, equipo de protección en el trabajo y no almacenar envases de plaguicidas en el hogar-, apuntan al mismo objetivo: evitar o disminuir el riesgo por exposición a POC's. Ahora bien, para enfatizar la necesidad de cambiar cierta(s) práctica(s) o conducta(s), se recomienda complementar el mensaje con dos variables ligadas al riesgo: "edad" y "susceptibilidad", por ejemplo:

“...No todas las etapas de la vida ni todas las personas son igualmente susceptibles a los efectos de las sustancias tóxicas contenidas en los residuos peligrosos..."

Este enunciado hace ver que no tan sólo es crítico conocer que la exposición puede constituir un riesgo, sino también el saber qué grupo de la población expuesta puede ser más susceptible al riesgo. Con tales conceptos integrados al mensaje se pretende que las personas se percaten e interesen por conocer:

1. Qué son esas sustancias llamadas POC's que se encuentran en su entorno.

2. Cuáles son las condiciones de exposición en las que los POC's pueden convertirse en un riesgo.

3. Cuáles son las medidas que deben seguir para prevenir esa situación de riesgo.

El o los conceptos integrados al mensaje deben ser claros, sencillos y precisos, evitar al máximo que sean ambiguos o confusos. Sin olvidar que el propósito es que la población objetivo capte un pensamiento básico: "más vale prevenir que remediar". El fín es concientizar, informar y propiciar un cambio de conducta para disminuir el riesgo por exposición a compuestos químicos. El mensaje se transmite "...como la probabilidad de riesgo por presencia de POC's... que se eleva con ciertas prácticas inadecuadas en su hogar y en el trabajo...". Reforzar que el riesgo es para él y su familia.

La forma adoptada para la transmisión del mensaje combina mensajes escritos, orales y visuales por considerar que todos se complementan y ayudan a alcanzar los propósitos de la estrategia de comunicación: aumentar la conciencia sobre el riesgo por POC's; informar sobre el riesgo por POC's; y cambiar la conducta ante el riesgo por POC's.

Las tres formas de mensajes elegidas han sido señaladas por el INESEMARNAP (2000: 81-85) como las más apropiadas de acuerdo a los objetivos que persigue esta investigación, a excepción de que en el objetivo "informar sobre el riesgo" se consideran también convenientes las "aplicaciones por computadora". Sin embargo, en el caso de localidades rurales, resultaría poco contundente por ser pocas las personas que cuentan con tal equipo o tienen acceso a internet. En Pesqueira, Sonora, aproximadamente 96 viviendas particulares disponen de computadora y son menos las 
que han contratado el servicio de internet. Es casi seguro que los jornaleros/as no disponen de tal equipo ni del servicio (cálculo propio con base en INEGI, 2010).

En todas las formas utilizadas para la transmisión del mensaje del riesgo (escrito, oral, visual) se cuida el lenguaje. La estrategia contempla un taller y varios materiales de apoyo (carteles, tríptico y video). Es importante señalar que el mensaje y el vocabulario utilizado en el material de apoyo y la información que se maneja en los talleres, se ajusta a los perfiles de la población destino (estudiantes de secundaria o preparatoria, jornaleros/as agrícolas y amas de casa). A la fecha, el acercamiento a la población de Pesqueira para explorar su percepción sobre la presencia y riesgos por POC's, ha mostrado que efectivamente están en riesgo, que desconocen tal hecho, que las prácticas de cuidado e higiene personal que llevan a cabo de manera cotidiana la mayoría de los jornaleros/as no son del todo adecuadas e incluso que acentúan esa situación de riesgo para ellos y su familia. Se confirma la necesidad de informar, sensibilizar y motivar a la población para que realice las prácticas de cuidado e higiene adecuadas en su vida cotidiana (hogar y trabajo). Lograr la eficiencia en la comunicación no es tarea fácil, hay que ser sistemáticos, constantes y comprometer en el camino al mayor número de actores e instituciones sociales posibles. La responsabilidad social de los empresarios agrícolas ayudaría bastante. Se trata de crear sinergia para que sea posible la obtención de mejores resultados.

Hoy por hoy, la buena noticia es que concretar una estrategia de comunicación con las características descritas tiene fines preventivos y no es en atención a una catástrofe o brote epidemiológico. Es el mejor momento de llevarla a cabo y de valorar incluso su proceso de implementación y resultados, todo con vistas a mejorar su efectividad. No esperemos a que sea demasiado tarde. 


\section{Bibliografía}

Aijón C.,y Cumplido A., (2007), "Percepción del riesgo de los agroquímicos en la localidad de Basavilbaso, Entre Ríos", Univ. Autónoma de Barcelona. Visto: 25 de junio de 2011. Disponible en: http://ddd.uab.cat/pub/trerecpro/ 2007/hdl_2072_5219/PFCAijon+Cumplido.pdf.

Beck, U. (1986), La Sociedad del Riesgo. Hacia una nueva modernidad. Paidós. Barcelona, España.

Ídem (2007), La Sociedad del Riesgo Mundial. En busca de la seguridad perdida. Paidós. Barcelona, España.

Bellingham, M., Fowler, P., Amezaga, M., Rhind, S., Cotinot, C., MandonPenin, B., Sharpe, R., y Evans, N. (2009), "Exposure to complex Cocktail of Environmental Endocrine-Disrupting Conpounds Disturbs the Kisspeptin/GPR54 System in Ovine Hypothalamus and Pituitary Gland", Environmental Health Perspective, 117(10), 1556-1552.

Camarena G. B. (2006), "La educación ambiental en el marco de los foros internacionales: una alternativa de desarrollo". Revista Estudios Sociales. Núm. 28, julio-diciembre. Págs. 7-42.

Carson L. Rachel (2010), Primavera silenciosa, Colección Drakontos Bolsillo. Editorial Crítica, Barcelona ( $1^{\mathrm{a}}$ edición 1962).

Codru, N., Schymura M., Negoita, S., Rej, R., y Carpenter, D. (2007), "Diabetes in Relation to Serum Levels of Polychlorinated Biphenyls and Chlorinated Pesticides in Adult Native Americans", Environmental Health Perspectives, 115(10), 1747-1752.

Echemendía, B. (2011), "Definiciones acerca del riesgo y sus implicaciones" en Rev Cubana Hig Epidemiol, Vol.49, No.3, Pp.470-481. Visto: 6 de febrero, 2011. Disponible en: http://scielo.sld.cu/pdf/hie/v49n3/hie14311.pdf.

EPA (2011), The U.S. Environmental Protection Agency (USEPA). List of minimum limitsof risk (MLR) for pesticides in soil. Visto: agosto, 2011.Disponible en: http://www.epa.gov/gateway/science/pesticides.html.

Fernández A., et al., (2005), Las sustancias tóxicas persistentes, Secretaría de Medio Ambiente y Recursos Naturales - Instituto Nacional de Ecología, Pp.19-20, México, http://siscop.ine.gob.mx/descargas/publicaciones/ sustancias_toxicas_persistentes.pdf

Giddens, A. (2000), Un mundo desbocado. Los efectos de la globalización en nuestras vidas. Ed. Taurus.

Gómez, Y. F. (2007), “Identificación y cuantificación del DDT y sus metabolitos en Leche Materna de mujeres Residentes de Pesqueira, Sono- 
ra". Universidad de Sonora, Departamento de Ciencias Químico-Biológicas, México, 92p.

Guillete L. J. Jr, Gross TS, Masson GR, Matter JM, Percival HF, Woodward AR. (1994), "Developmental abnormalities of the gonad and abnormal sex hormone concentrations in juvenile alligators form contaminated and control lakes in Florida", Environ Health Perspect, 102: 680-688.

Hazardous Chemicals Conventions (2002). en Fernández A.; Yarto, M.; y Castro, J.; (2005), Las sustancias tóxicas persistentes. Secretaría de Medio Ambiente y Recursos Naturales - Instituto Nacional de Ecología, Pp. 21. México. Disponible en: http://siscop.ine.gob.mx/descargas/publicaciones/ sustancias_toxicas_persistentes.pdf

IPEN (2011), Working together for a toxic-free future. International POP's Elimination Network. http://www.ipen.org/ipenweb/generalpublic/ whatpops.html. [Visto: 3 de mayo, 2011].

INE-SEMARNAP (2000), Características de peligrosidad ambiental de plaguicidas. Promoción de la prevención y reducción de riesgos químicos ambientales. http://www2.ine.gob.mx/publicaciones/pclave_t_res.php. [Visto: 15 de enero, 2012].

INE-SEMARNAP (2000), Comunicación de riesgos para el manejo de sustancias peligrosas con énfasis en residuos peligrosos, http:// www2.ine.gob.mx/publicaciones/pclave_t res.php [Visto: 15 de enero, 2012].

INEGI (2010), Conteo de Población y Vivienda 2010. Visto: 9 Diciembre 2011.Disponible en: http://www3.inegi.org.mx/sistemas/iter/ entidad_indicador.aspx?ev $=5$.

Lee, D.H., Steffes M., Jacobs D. (2007), "Possitive Associations of Serum Concentrations of Polychclorinated Biphanyls or Organochlorine Pesticides with Self-Reported Arthritis, Especially Rheumatoid Type, in Woman", Environmental Health Perspective, 115(6), 883-888.

Martínez, C., y Gómez, S. (2007), "Riesgo genotóxico por exposición a plaguicidas en trabajadores agrícolas", Revista Internacional de Contaminación Ambiental, 23(4), 185-200.

Ochoa, B. et al. (2012), "Situación de riesgo por la presencia de Contaminantes Orgánicos Persistentes: normatividad, problemática e impactos locales”. En Revista de Ciencias Sociales. 2012.

Ohlendorf HM, Swineford DM, Locke LN. (1993), “Organochlorine poisoning of herons". In: Proceedings of the Conference of the Colonial Waterbird Group, Vol. 3 (Southern WE, et al., eds), Lafayette, Lousiana. DeKalb, IL:North Illionois University, 176-185. 
Organización Panamericana de la Salud (2003), Curso de Autoinstrucción: Comunicación de Riesgos (curso en línea). Oficina Regional de la Organización Mundial de la Salud [En línea] Área de Desarrollo Sostenible y Salud Ambiental. Visto: 14 de mayo de 2011. Disponible en: http:// www.opas.org.br/ambiente/risco/tutorial6/e/temas.html

PNUMA (2008), Convención de Estocolmo, Suiza. Programa de las Naciones Unidas para el Medio Ambiente. http:/chm.pops.int/Convention/ The\%20POPs/tabid/673/language/en-US/Default.aspx [Visto: 16 junio, 2010].

PNUMA (2010). “Convenio de Estocolmo sobre los contaminantes orgánicos persistentes (COP). Los nuevos 9 COPs". Programa de las Naciones Unidas para el Medio Ambiente. [En línea] Ginebra, Suiza pp.1-15. Visto: 16 de febrero de 2012. Disponible en: www.pops.int.

Rantakokko, P., Kiviranta, H., Rylander, R., Hydbom, A., y Vartiainen, T. (2009), "A simple fast liquid-liquid extraction method for determination of 2,2',4, 4',5, 5'-hexachlorobiphenil (CB-153) and 1,1'.dichloro-2,2'-bis (ochlorophenyl)-ethylene (p, p'-DDE) from human serum for epidemiological studies of type 2 diabetes", Journal of Cromatography A, 12(16), 807901.

Roberts, E., English, P., Grether, J., Windham, G., Somberg, L., y Wolff, C. (2007), "Maternal Residence near agricultural pesticide aplications and autism spectrum disorders among children in California Central Valley", Environmental Health Perspectives 15(10): 1482.

Sagiv, S. Nugent, J., Brazelton, T., Choi, A., Tolbert, P., Altshul, L., y Korrick, S. (2008), "Prenatal Organochlorine Exposure and Measures of Behavior in Infancy Using the Neonatal Behavioral Assesment Scale (NBAS)", Environmental Heatlh Perspectives, 116(5), 666-673.

Sánchez, J. (2001), Salud y medio ambiente. Una perspectiva Sociológica. http://rua.ua.es/dspace/bitstream/10045/2725/7/cap7.pdf. 8-11 [visto: marzo 5, 2011].

SEMARNAT-INE-CONACYT, Fondo Sectorial de Investigación Ambiental, Convocatoria 2008. Fondo S0010-2008-1, modalidad a1, http:// www.conacyt.mx/Fondos/Sectoriales/SEMARNAT/2008-01.

Silveira, M. et al. (2011), Valoración del riesgo de exposición a insecticidas organofosforados en adultos del sexo masculino en Sonora, México. CIENCIA@UAQ. [En línea] Vol.4, No.2., Pp.70-81. Visto: 13 de febrero, 2012. Disponible en: http:/www.uaq.mx/investigacion/revista_ciencia@uaq/ ArchivosPDF/v4-n2/t8.pdf.

Torres, L., y López L, (2007), “Efectos a la salud y exposición a p, p'-DDE y p, p'-DDT el caso México”, Ciencia e Saúde Colectiva. 12(001), 51-60. 
Valenzuela, A., et al. (2008), Evaluación Directa a Plaguicidas de Jornaleros Agrícolas e Indirecta por Consumo de Agua y Alimentos y su Impacto en la Expresión del Síndrome Metabólico. Informe Técnico FinalCONACYT.

Valenzuela, A., et al. (2010), Desarrollo de una metodología que permita llevar a cabo el inventario, caracterización y verificación de sitios potencialmente contaminados con compuestos orgánicos persistentes en las principales zonas agrícolas del estado de Sonora. Proyecto 108092 financiado por Fondo Sectorial de Investigación Ambiental, SEMARNAT-INECONACYT, Convocatoria 2008. Fondo S0010-2008-1. Publicación de Resultados 23 marzo 2010. http://www.conacyt.mx/Fondos/Sectoriales/ SEMARNAT/2008-01/Semarnat_Resultados_2008-01.pdf, marzo 23, 2010. Fondo S0010, convocatoria $\bar{S} 0010-2008-\overline{1}$, modalidad a1.

Walker C. H., S. P. Hopkin, R. M. Sibly, and D.B. Peakall (1996), "Biomarkers in population studies". In Walker C. H. et al., Principles of ecotoxicology, Taylor \& Francis. Bristol, U.K.

Wang, X., Wang, D., Quin, X., y Xu., X. (2008), "Residues of organochlorine pesticides in surface soils from college school yards in Beijing, China", Journal Of Environmental Sciences, 20, 1090-1096.

Ward, M., Colt, J., Metayer, C., Gunier, R., Lubin, J., Crouse, V., Nishioka, M., Reynolds, P., y Buffler, P. (2009), "Residential Exposure to Polychlorinated Biphenyls and Organochlorine Pesticides and Risk of Childhood Leukemia”, Environmental Health Perspectives, 117 (6):100710013.

Xu, X., Dailey A., Talbott E., Ilacqua V., Kearney G., y Asal N. (2010), "Associations of Serum Concentrations of Organochlorine Pesticides with Breast Cancer and Prostate Cancer in U. S. Adults", Environmental Health Perspectives, 118(1), 60-66.

Yassi A, et al. (2000), Salud Ambiental Básica. Unidad de Producciones Gráficas del MINREX, La Habana. 